\title{
The Be/X-ray transient HD34921
}

\author{
V. F. Polcaro ${ }^{1}$, L. Norci ${ }^{2}$, E. J. A. Meurs ${ }^{3}$, S. Bernabei ${ }^{4}$ and \\ A. S. Miroshnichenko ${ }^{5}$ \\ ${ }^{1}$ IASF-INAF, Rome, Italy \\ email: polcaro@rm.iasf.cnr.it \\ ${ }^{2}$ School of Physics, Dublin City University, Dublin, Ireland \\ ${ }^{3}$ Dunsink Observatory, Dublin Institute for Advanced Studies, Castleknock, Dublin 15, Ireland \\ ${ }^{4}$ Osservatorio Astronomico, Bologna, Italy \\ ${ }^{5}$ University of Toledo, USA
}

\begin{abstract}
HD34921 has been identified as the counterpart of the X-ray source 4U0515+38 $(=1 \mathrm{H} 0521+373)$. The InfraRed properties are reminiscent of the B[e] system CI Cam. Optical short-term variability suggests a compact companion. We discuss how this system fits in the overall framework of Be stars and X-ray binaries.
\end{abstract}

Keywords. Stars: B[e], Stars: X-rays, Stars: Neutron

HD34921 $\left(\mathrm{BD}+37^{\circ} 1160\right)$ was identified as counterpart of the X-ray source $4 \mathrm{U} 0515+38$ by Polcaro et al. (1990; P90). After the original Uhuru observations, this X-ray transient was detected again by HEAO1 as $1 \mathrm{H} 0521+373$, at a much lower flux level. Subsequent balloon-borne hard X-ray telescopes also detected this source (at still higher energies), in 1980 and 1981 (Ubertini et al. 1982; Polcaro et al. 1984). Following upper limits with EXOSAT's ME detectors (P90), 4U0515+38 has not been seen anymore at high energies. Data for the 2-7 keV band are shown in Figure 1.

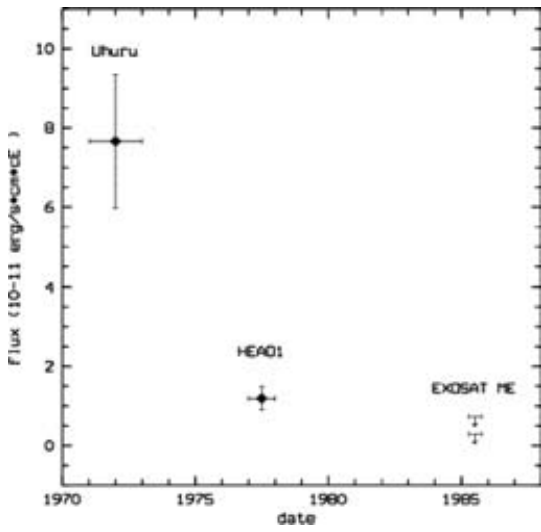

Figure 1. X-ray lightcurve of HD34921 at 2-7 keV.

The optical counterpart, HD 34921, is a known variable star. P90 classified it as B0III-IVpe and estimated a distance of $1.7 \mathrm{kpc}$. A number of spectroscopic studies has been carried out since its association with the X-ray source $4 \mathrm{U} 0515+38$.

Spectra taken in 1990 show a variable HeII $\lambda 4686$ emission line, which is a recognized indicator of an accretion disk and led to the identification of HD 34921 as the counterpart of 4U0515+38 (P90). Classical Be stars do not have HeII in emission, while it is present in other X-ray transients like $\mathrm{X}$ Per and $\gamma$ Cas. At the same time, the emission lines of $\mathrm{HeII}$ and $\mathrm{H}_{\alpha}$ as well as $\mathrm{H}_{\beta}$ were found to vary on short timescales, of order of $300 \mathrm{~s}$ for $\mathrm{V} / \mathrm{R}$ (violet/red wing) variations (Rossi et al. 1991). Both $\mathrm{H}_{\alpha}$ and $\mathrm{H}_{\beta}$ were split, as is typical for a disk structure, and since the same variability timescale is observed for HeII (which points at an accretion disk), the split $\mathrm{H}_{\alpha}$ and $\mathrm{H}_{\beta}$ profiles are thought to refer similarly to the surroundings of a compact object. We note that variability due to e.g. hotspots in a Be star (excretion) disk would have timescales of months. $\mathrm{H}_{\alpha}$ and (permitted) FeII emission lines, varying in phase with $\mathrm{H}_{\beta}$, have been seen split on occasions as well. 
Spectroscopic data taken in 2003 (Polcaro et al., in preparation), suggest a third, nonvariable component in the $\mathrm{H}_{\beta}$ profiles. Polcaro et al. (2005) infer that this component also filled in the top of the $\mathrm{H}_{\alpha}$ profile in 2003 due to a second emission region besides the accretion disk, which presumably features lower temperature and higher density than the disk. Periods of high Equivalent Width and non-split $\mathrm{H}_{\alpha}$ may signify shell ejection phases.

In the spectral energy distribution (SED) of HD 34921 a Near-InfraRed excess has been observed in the J, H, K bands (P90) that appears as a thermal free-free component, which has been interpreted as an extended atmosphere. The Far-InfraRed source IRAS $051921+3737$ has also been associated with this star (P90), suggesting a very cold dust envelope, which is uncommon for HMXBs. Clark et al. (1999) noticed strong analogies between the Near-InfraRed spectra of HD 34921 and CI Cam, confirming a very complex circumstellar environment. As for CI Cam, these authors proposed a B $[\mathrm{e}]$ classification also for HD 34921, based on the observed analogies in the InfraRed. The two stars would be the only X-ray binary counterparts of the B[e] spectral type.

Considering the B[e] classification criteria adopted by Lamers et al. (1998), in HD34921 the required forbidden emission lines of [FeII] and [OI] in the optical spectrum are absent, while rather than a strong Near-/Mid-IR excess due to hot circumstellar dust it features a cold dust envelope. Therefore, the B[e] classification of HD34921 is subject to some doubt (Norci et al. 2006).

The absence of forbidden lines in the optical spectrum of HD34921 means that the circumstellar environment has (regions of) high density. This could be e.g. ring-shaped shell ejections. In CI Cam only forbidden emission lines are seen produced in the envelope, while the photospheric lines are not visible (unlike HD34921). The observation that in HD34921 also non-Balmer, permitted emission lines follow the 300 seconds variation suggests that these too are linked with the probable accretion disk, rather than with a stellar excretion disk, for which there is little direct evidence.

We note that HD34921 is an OB star, outside an association. The proper motions correspond to a velocity of $42 \mathrm{~km} / \mathrm{s}$, which qualifies HD34921 as a runaway star. The high velocities of OB runaway stars are thought to have been caused by either dynamical ejection from a dense cluster (thus in the early stages of development of OB associations) or a supernova event in a binary system. In the case of HD 34921, where there is evidence for a collapsed companion, the supernova in a binary mechanism provides the likely explanation for the high velocity (and the system survived the supernova event, as a good fraction of systems are likely to do; see also O'Maoileidigh et al. 2005). Thus, binary evolution has likely played a role in the evolution of HD 34921. Due to the presence of a companion, outer disk parts (with lesser density) may become truncated due to Roche lobe cut-off, which interestingly provides an other way to suppress forbidden line emission.

\section{References}

Clark, J.S., Steele, I.A., Fender, R.P. \& Coe, M.J. 1999, A\&A 348, 888

Lamers, H.J.G.L.M., Zickgraf, F.-J., de Winter, D., et al. 1998, A\&A 340, 117

Norci, L., et al. 2006, ASP Conf. Series, in press

O'Maoileidigh, C., Meurs, E.J.A. \& Norci, L. 2005, NewA 10, 591

Polcaro, V.F., et al. 1984, A\&A 131, 229

Polcaro, V.F., et al. 1990, A\&A 231, 354 (P90)

Polcaro, V.F., Norci, L., Meurs, E.J.A. \& Bernabei, S. 2005, in press

Rossi, C., Norci, L. \& Polcaro, V.F. 1991, A\&A 249, L19

Ubertini, P., et al. 1982, A\&A 106, 174 